\title{
Some Remarks on Quasi-Invariant Actions of Loop Groups and the Group of Diffeomorphisms of the Circle
}

\author{
Yurii A. Neretin \\ Chair of Analysis, MIEM (Moscow Institute of Electronis and Mathematics), \\ Bolskoy Vuzovskii 3/12, Moscow 109028, Russia
}

Received: 15 September 1993

\begin{abstract}
We construct the series of quasi-invariant actions of the group Diff of diffeomorphisms of the circle and loop groups on the functional spaces provided by non-Wiener Gauss measures. We construct some measures which can be considered as analogues of Haar measure for loop groups and the group Diff. These constructions allow us to construct series of representations of these groups including all known types of representations (highest weight representations, energy representations, almost invariant structures, etc.)
\end{abstract}

\section{Introdution}

In $[\mathrm{N} 1, \mathrm{~N} 2]$ there were constructed some series of quasi-invariant actions of the group of diffeomorphisms of the circle and loop groups on functional spaces with (nonWiener) Gauss measures. In this paper we use results of [N1, N2] for constructing some "new" dynamical systems for loop groups and the group of diffeomorphisms of the circle. The paper also contains some results which are interesting for representation theory and the theory stochastic processes.

Let Diff ${ }^{\infty}$ denote the group of $C^{\infty}$-smooth preserving orientation diffeomorphisms of the circle $S^{1}=\mathbb{R} / 2 \pi \mathbb{Z}$. Let $K$ be compact Lie group. We denote by $\mathbb{L}^{\infty}(K)$ the group of $C^{\infty}$-smooth functions $S^{1} \rightarrow K$. We also define the groups Diff ${ }^{1}$ of orientation preserving diffeomorphisms of class $C^{1}$ and the groups $\mathbb{L}^{0}(K)$ of functions $S^{1} \rightarrow K$ of class $C^{0}$.

In this paper we construct the following dynamical systems:

- the series of Diff ${ }^{\infty}$-quasi-invariant measures on the space $\operatorname{Diff}^{1} / \mathbb{T}$, where $\mathbb{T}$ is the group of rotations of the circle,

- the series of Diff ${ }^{\infty}$-quasi-invariant measures on Diff ${ }^{1}$,

- Diff ${ }^{\infty}$-quasi-invariant measures on the space of Cantor subsets of the circle.

- $\mathbb{L}^{\infty}(S O(n))$-quasi-invariant measures on $\mathbb{L}^{0}(S O(n))$. These measures are also Diff $^{\infty}$-quasi-invariant 
- $\mathbb{L}^{\infty}(S O(n))$-quasi-invariant measures on spaces of maps $S^{1}$ to homogeneous spaces $S O(n) / H$.

Now we will briefly discuss the influence of these constructions to representation theory. Since 1970 there were the following attempts to construct representation theory for the groups Diff ${ }^{\infty}$ and $\mathbb{L}^{\infty}(K)$.

1. The most well-known is highest weight representation theory (see [Ka, PS]).

2. Representations of Diff ${ }^{\infty}$ of finite functional dimension and the attempts to apply the orbit method (see for instance [Ki]).

3. Ismagilov's paper [I].

4. "Energy representations" of $\mathbb{L}^{\infty}(K)$ connected with the Wiener measure (Ismagilov, Vershik, Gelfand, Graev, Albeverio, Testard, Hoegh-Krohn, I. Frenkel, Malliavin, Gross, see [AHTV]).

5. "Almost invariant structures" (Neretin, see [N1-N6]).

None of the specialists (including the author) believed that it is possible to unite these five theories, it seemed that here we had the objects of essentially different nature. This paper doesn't contain much representation theory. But as the result of this paper there is union of all approaches 1)-5).

\section{Invariant Measure on Hilbert Space}

This section contains brief survey of classical results (I. Segal, Feldman, Hajek, Prokhorov, Sazonov, L. Gross, etc.) on measures in Hilbert spaces (for details see the books of Kuo [Kuo] or Shilov, Fan Dyk Tin [ShF]).

\subsection{The Canonical Extension for $\ell_{2}$}

Let us consider the real line $\mathbb{R}$ with the Gauss measure $d \mu=\frac{1}{\sqrt{2 \pi}} e^{-x^{2} / 2} d x$. Denote by $\mathbb{R}^{\infty}$ the product of infinite (countable) number of copies of $\mathbb{R}$ provided by the product $\nu$ of the measures $d \mu$.

Proposition 1.1. $\ell_{2}$ has zero measure in $\mathbb{R}^{\infty}$.

Theorem 1.1. Let $\left(t_{1}, t_{2}, \ldots\right) \in \ell_{2},\left(x_{1}, x_{2}, \ldots\right) \in \mathbb{R}^{\infty}$. Then for fixed $\left(t_{1}, t_{2}, \ldots\right)$ the series $\sum t_{j} x_{j}$ converges almost everywhere on $\mathbb{R}^{\infty}$.

Now let us consider infinite real orthogonal matrix $A$. By Theorem 1.1 for almost all $x=\left(x_{1}, x_{2}, \ldots\right)$ the vector

$$
A x=\left(\begin{array}{cc}
a_{11} & a_{12} \cdots \\
a_{21} & a_{22} \cdots \\
\ldots
\end{array}\right)\left(\begin{array}{c}
x_{1} \\
x_{2} \\
\ldots
\end{array}\right)=\left(\begin{array}{c}
a_{11} x_{1}+a_{12} x_{2}+\ldots \\
a_{21} x_{1}+a_{22} x_{2}+\ldots \\
\ldots
\end{array}\right)
$$

is well-defined.

Theorem 1.2. For each real orthogonal matrix $A$ the map $x \mapsto A x$ preserves the measure $\nu$ on $\mathbb{R}^{\infty}$.

Let us denote by $O(\infty)$ the group of all infinite real orthogonal matrixes.

Remark. We saw that the group $O(\infty)$ acts on the space $\mathbb{R}^{\infty}$ with the measure $\nu$. Here $\mathbb{R}^{\infty}$ is the space with measure, the group $O(\infty)$ doesn't act on the set $\mathbb{R}^{\infty}$ ! 
Theorem 1.3. Let $h \in \ell_{2}$. Then the measure $\nu$ is quasi-invariant with respect to transformations $x \mapsto x+h$.

\subsection{The Canonical Extension for Arbitrary Hilbert Space}

Let $H$ be real Hilbert space. Let $e_{1}, e_{2}, \ldots$ be orthogonal basis in $H$. Then we can identify $H$ and $\ell_{2}$, the space $\ell_{2}$ is a subset in $H$, and so we have embedding $H \cong \ell_{2} \rightarrow \mathbb{R}^{\infty}$. Extension $\hat{H}$ of $H$ is, by definition $\mathbb{R}^{\infty} \supset H$.

It seems that the construction depends on a basis in $H$. Let $e_{1}, e_{2}, \ldots$ and $f_{1}, f_{2}, \ldots$ be two orthogonal bases in $H$, let us denote the corresponding extensions by $\hat{H}\left(e_{1}, e_{2}, \ldots\right)$ and $\hat{H}\left(f_{1}, f_{2}, \ldots\right)$. Let $A$ be an orthogonal operator in $H$ such that $A e_{\imath}=f_{i}$. Then the map $x \mapsto A x$ from $\hat{H}\left(e_{1}, e_{2}, \ldots\right)$ to $\hat{H}\left(f_{1}, f_{2}, \ldots\right)$, is the isomorphism of the spaces with measures. We see that $\hat{H}\left(e_{1}, e_{2}, \ldots\right)$ and $\hat{H}\left(f_{1}, f_{2}, \ldots\right)$ are canonically isomorphic and hence our construction doesn't depend on choice of a basis.

\subsection{Gross Construction of the Canonical Extension}

Theorem 1.4. Let $\Lambda=\left(\lambda_{1}, \lambda_{2}, \ldots\right)$ be a sequence of real positive numbers and $\sum \lambda_{j}^{2}<\infty$. Let $\Omega_{\Lambda}$ be the set of all $x=\left(x_{1}, x_{2}, \ldots\right) \in \mathbb{R}^{\infty}$ such that $\sum \lambda_{j}^{2} x_{j}^{2}<\infty$. Then the measure of the set $\mathbb{R}^{\infty} \backslash \Omega_{\Lambda}$ equals zero.

Embedding $\Omega_{\Lambda} \rightarrow \mathbb{R}^{\infty}$ is an isomorphism of spaces with measure and so we can replace the space $\mathbb{R}^{\infty}$ with $\Omega_{\Lambda}$. Thus $\Omega_{\Lambda}$ can be looked upon as the canonical extension $\hat{\ell}_{2}$ of $\ell_{2}$. It is important to notice that the space $\Omega_{\Lambda}$ is the Hilbert space, the scalar product in $\Omega_{\Lambda}$ is given by the formula

$$
\left\{\left(x_{1}, x_{2}, \ldots\right),\left(y_{1}, y_{2}, \ldots\right)\right\}=\sum \lambda_{\jmath}^{2} x_{\jmath} y_{j} .
$$

Let us describe the same construction on invariant language. Let $H$ be real Hilbert space with scalar products $\langle\cdot, \cdot\rangle$, let $A: H \rightarrow H$ be the Hilbert-Schmidt operator, let $A^{*} h \neq 0$, if $h \neq 0$. We define in $H$ a new scalar product

$$
\{x, y\}=\langle A x, A y\rangle \text {. }
$$

Let $K$ be the completion of $H$ relative to the norm $\|x\|=\{x, x\}^{1 / 2}$. It is easy to see that the operator $A: H \rightarrow H$ extends to the well-defined (unitary) operator $A: K \rightarrow H$. The probabilistic measure $\nu$ in $K$ is defined by the equality

$$
\int_{K} \exp (i\{x, y\}) d \nu(y)=\exp (-\{A x, A x\}) .
$$

Then it turns out to be $K \cong \hat{H}$. To see this let us consider the orthogonal bases $e_{1}, e_{2}, \ldots$ and $f_{1}, f_{2}, \ldots$ in $H$ such that $A e_{i}=\lambda_{i} f_{\imath}\left(\lambda_{j}>0\right)$. Let us identify $H$ and $\ell_{2}$ using the basis $e_{1}, e_{2}, \ldots$ and embed $\ell_{2}$ to $\mathbb{R}^{\infty}$. Then the space $K$ is identified with the space $\Omega_{\Lambda}$

$$
K \ni \sum x_{i} f_{i} \leftrightarrow\left(x_{1}, x_{2}, \ldots\right) \in \Omega_{\Lambda} .
$$

The space $K$ is named Hilbert-Schmidt extension of $H$.

Proposition 1.2. Let $h \in H$. Then the measure $\nu$ on $K$ is quasi-invariant with respect to the translation $x \mapsto x+h$. 


\subsection{The Feldman-Hajek Theorem}

Let $H$ be real Hilbert space. Let us denote by $G L O(H)$ the group of all operators $A: H \rightarrow H$ of the form $A=B(1+T)$, where $B$ is the orthogonal operator and $T$ is a Hilbert-Schmidt operator.

Lemma 1.1. Let $A$ be an operator in $H$. Then the following conditions are equivalent:

1. $A \in G L O(H)$,

2. $A^{*} A-E$ is Hilbert-Schmidt operator.

Now let $A$ be bounded operator in $H$. Let us identify $H$ and $\ell_{2}$. Then the map $x \mapsto A x$ [see (1.1)] is defined almost everywhere.

Theorem 1.5. Let $A \in G L O(H)$. Then the canonical measure in $\hat{H}$ is quasi-invariant with respect to the map $x \mapsto A x$.

Remark. Of course, the group $G L O(H)$ doesn't act on the set $\hat{H}$, it acts on the space $\hat{H}$ with measure.

Let $H$ be a real Hilbert space, $A: H \rightarrow H$ be a Hilbert-Schmidt operator, let $K$ be the corresponding Hilbert-Schmidt extension of $H$. Let $G L O(H \mid K)$ be the group of bounded invertible linear operators $A$ in $K$ such that

a) $h \in H$ implies $A h \in H$.

b) The restriction of $A$ to (dense) subspace $H$ is the element of the group $G L O(H)$ (with respect to scalar product in $H$ !)

The group $G L O(H \mid K)$ acts on the Hilbert space $K(x \mapsto A x$, it is the usual linear operator and so $A: K \rightarrow K$ is the map of sets!) and the canonical measure on $K$ is quasi-invariant with respect to this action.

\subsection{Space $L^{2}(\hat{H})$ as Bosonic Fock Space}

Let $O(H)$ be the group of all orthogonal operators in the space $H$. Define the representation

$$
T(A) f(x)=f(A x)
$$

of $O(H)$ in $L^{2}(\hat{H})$. We want to describe the decomposition of this representation.

Let $H=\ell_{2}$. Let us consider the Hermite polynomials $h_{0}(x), h_{1}(x), h_{2}(x), \ldots$ :

$$
\frac{1}{\sqrt{2 \pi}} \int h_{j}(x) h_{k}(x) e^{-x^{2} / 2} d x=\delta_{\jmath, k}
$$

(degree of $h_{j}(x)$ equals $j$ ).

Let $R_{K} \subset L^{2}\left(\mathbb{R}^{\infty}\right)$ be the set of linear combinations of all polynomials

$$
h_{i_{1}}\left(x_{1}\right) h_{i_{2}}\left(x_{2}\right) \ldots ; \quad i_{1}+i_{2}+\ldots=K .
$$

Proposition 1.3. a) $L^{2}\left(\mathbb{R}^{\infty}\right)$ is the orthogonal sum

$$
L^{2}\left(\mathbb{R}^{\infty}\right)=\bigoplus_{j \geq 0} R_{j} .
$$

b) The restriction of the representation $T$ on $R_{j}$ is irreducible and equivalent to the $j$-th symmetric power of the identical representation of $O(\infty)$ in $\ell_{2}$. 
Remark. The direct sum of all symmetric powers of a Hilbert space is named the bosonic Fock space and hence $L^{2}(\hat{H})$ is one of the models of the bosonic Fock space.

\section{The Basic Construction}

This section contains an account of the results of the papers [N1, N2] (see also [N5, N6]).

\subsection{Sobolev Spaces on the Circle}

Let us denote by $W^{s}$ the Sobolev space, i.e. the space of all (generalized) real functions $f(\varphi)=\sum c_{n} e^{i n \varphi}$ on the circle such that

$$
\sum\left(n^{2}+1\right)^{s}\left|c_{n}\right|^{2}<\infty .
$$

The scalar products in $W^{s}$ is given by the formula

$$
\left\langle\sum c_{n} e^{i n \varphi}, \sum d_{n} e^{i n \varphi}\right\rangle=\sum\left(n^{2}+1\right)^{s} c_{n} \bar{d}_{n} .
$$

Remarks. 1. The space $W^{0}$ is the usual $L^{2}\left(S^{1}\right)$.

2. If $s<0$ then elements of $W^{s}$ are generalized functions.

3. If $s>1 / 2$ then functions $f \in W^{s}$ are continuous.

4. If $s \geq 1$ then functions $f \in W^{s}$ are differentiable almost everywhere.

\subsection{The Basic Construction for Diff ${ }^{\infty}$}

Let Diff $^{\infty}$ act in $W^{s}$ by transformations

$$
T_{s}(q) f(\varphi)=f(q(\varphi)) q^{\prime}(\varphi)^{1 / 2-s} .
$$

Theorem 2.1. $T_{s}(q) \in G L O\left(W^{s}\right)$.

This theorem gives us the series of quasi-invariant actions of Diff ${ }^{\infty}$ on the canonical extensions of $W^{s}$. The case $s=1$ corresponds to the usual Wiener measure (see p. 2.4).

\subsection{The Basic Construction for $\mathbb{L}^{\infty}(S O(m))$}

Let $S O(m)$ be the group of real orthogonal operators in $\mathbb{R}^{m}$. Let $\mathbb{L}^{\infty}(S O(m))$ be the group of smooth functions $S^{1} \rightarrow S O(m)$. Denote by $W_{m}^{s}$ the space of real-valued functions $F=\left(f_{1}, f_{2}, \ldots, f_{m}\right): S^{1} \rightarrow \mathbb{R}^{m}$ such that $f_{\jmath} \in W^{s}$ provided by the scalar product

$$
\left(F^{(1)}, F^{(2)}\right)_{s}=\sum_{J}\left\langle f_{j}^{(1)}, f_{j}^{(2)}\right\rangle_{s} .
$$

The group $\mathbb{L}^{\infty}(S O(m))^{\jmath}$ acts in $W_{m}^{s}$ by the transformations

$$
Q(A(\varphi)) F(\varphi)=A(\varphi) F(\varphi),
$$

where $A(\varphi) \in \mathbb{L}^{\infty}(S O(m))$. 
Theorem 2.2. $Q(A(\varphi)) \in G L O\left(W_{m}^{s}\right)$.

Proofs of Theorems. See Sects. 2.5-2.10.

Now let us notice that the group Diff ${ }^{\infty}$ acts on $\mathbb{L}^{\infty}(S O(m))$ by automorphisms

$$
A(\varphi) \mapsto A(q(\varphi)),
$$

where $q \in \operatorname{Diff}^{\infty}, A(\varphi) \in \mathbb{L}^{\infty}(S O(m))$. So we can consider the semidirect product $\operatorname{Diff}^{\infty} \ltimes \mathbb{L}^{\infty}(S O(m))$.

Let us construct the action of the group Diff ${ }^{\infty} \propto \mathbb{L}^{\infty}(S O(m))$ in the space $W_{m}^{s}$. The group $\mathbb{L}^{\infty}(S O(m))$ acts by the formula (2.3) and the group Diff ${ }^{\infty}$ acts by transformations

$$
T_{s}(q) F(\varphi)=q^{\prime}(\varphi)^{1 / 2-s} F(\varphi) .
$$

Corollary. We obtained the embedding

$$
\operatorname{Diff}^{\infty} \ltimes \mathbb{L}^{\infty}(S O(m)) \rightarrow G L O\left(W_{m}^{s}\right)
$$

\subsection{The Canonical Extension of $W^{s}$}

Lemma 2.1. Let $s_{1}-s_{2}>1 / 2$. Then the identical embedding $W^{s_{1}}$ to $W^{s_{2}}(f \mapsto f)$ is the Hilbert-Schmidt operator.

Proof. Evident.

Hence $W^{s-1 / 2-\varepsilon}$ can be looked upon as the canonical extension $\hat{W}^{s}$ of $W^{s}$ (where $\varepsilon>0)$.

Corollary 1. Let $q \in \operatorname{Diff}^{\infty}$. Then

$$
T_{s}(q) \in G L O\left(W^{s} \mid W^{s-1 / 2-\varepsilon}\right)
$$

Proof is evident, the operator $T_{s}(q)$ is bounded in each space $W^{\lambda}$.

Corollary 2. Let $A(\varphi) \in \mathbb{L}^{\infty}(S O(m))$. Then

$$
Q(A(\varphi)) \in G L O\left(W_{m}^{s} \mid W_{m}^{s-1 / 2-\varepsilon}\right) .
$$

Let us discuss the case $s=1$. The space $W^{1 / 2-\varepsilon}$ can be looked upon as the canonical extension of $W^{1}$. For instance $L^{2}\left(S^{1}\right) \cong W^{0}$ can be looked upon as the canonical extension $W^{1}$ of $\hat{W}^{1}$. It is well.known (see [Ku]) that the canonical measure on $L^{2}\left(S^{1}\right) \cong W^{0}$ is the usual Wiener measure. The support of the Wiener measure is contained in the space $C\left(S^{1}\right)$ of continuous functions on $S^{1}$ and so $C\left(S^{1}\right)$ can be looked upon as the space $\hat{W}^{1}$.

Corollary. The Wiener measure in $C\left(S^{1}\right)$ is quasi-invariant with respect to transformations

$$
f(\varphi) \mapsto f(p(\varphi)) p^{\prime}(\varphi)^{-1 / 2}
$$

where $p \in \operatorname{Diff}^{\infty}$. 


\subsection{Strongly Equivalent Scalar Products}

Let $H$ be Hilbert space with scalar product $\langle\cdot, \cdot\rangle$. Let $\{\cdot, \cdot\}$ be another scalar product in $H$. We say that $\langle\cdot, \cdot\rangle$ and $\{\cdot, \cdot\}$ are strongly equivalent if there exist $C>0$ and a Hilbert-Schmidt operator $T$ in $H$ such that

$$
\{x, y\}=C \cdot\langle(1+T) x, y\rangle .
$$

Now we want to replace the scalar products $(2.1)$ in the spaces $W^{s}$ to strongly equivalent scalar products which are more convinient.

A) Let $s$ be nonnegative integer, $s=n$. Suppose

$$
B_{n}(f, g)=\sum_{j=0}^{n-1} f^{(j)}(0) g^{(j)}(0)+\int_{0}^{2 \pi} f^{(n)}(\varphi) g^{(n)}(\varphi) d \varphi .
$$

Lemma 2.2. The scalar products $\langle\cdot, \cdot\rangle_{n}$ and $B_{n}(f, g)$ in $W^{n}$ are strongly equivalent. Proof. Evident.

B) $s=k+1 / 2$, where $k$ is nonnegative integer. Let

$$
\begin{aligned}
B_{K+1 / 2}(f, g)= & \sum_{j=0}^{K} f^{(j)}(0) g^{(\jmath)}(0) \\
& +\int_{0}^{2 \pi} \int_{0}^{2 \pi} \operatorname{ctg}\left(\frac{\varphi-\psi}{2}\right) f^{(k)}(\varphi) g^{(k+1)}(\psi) d \varphi d \psi .
\end{aligned}
$$

Lemma 2.3. a) $B_{k+1 / 2}(\cdot, \cdot)$ is the scalar product in $W^{k+1 / 2}$.

b) Scalar products $B_{k+1 / 2}(\cdot, \cdot)$ and $\langle\cdot, \cdot\rangle_{K+1 / 2}$ are strongly equivalent.

Proof. It is easy to see that

$$
\frac{1}{\pi} \int_{0}^{2 \pi} \operatorname{ctg}\left(\frac{\varphi-\psi}{2}\right) e^{i n \psi} d \psi=\operatorname{sg} n(n) e^{i n \varphi} .
$$

Now the lemma is evident.

c) $2 s \notin \mathbb{Z}$. Let us consider the following bilinear form in the space $C^{\infty}\left(S^{1}\right)$ of real smooth functions

$$
B_{s}(f, g)=\int_{0}^{2 \pi} \int_{0}^{2 \pi}\left|\sin \left(\frac{\varphi-\psi}{2}\right)\right|^{-1-2 s} f(\varphi) g(\psi) d \varphi d \psi .
$$

It is a well-known invariant form in the representation theory of $S L_{2}(\mathbb{R})$ (see [GGV]) and we will discuss some of its properties.

a) The integral (2.5) is convergent if $s<0$. In this case

$$
B_{s}\left(e^{i n \varphi}, e^{-i m \varphi}\right)=c_{n}(s) \delta_{n, m}
$$

where

$$
c_{n}(s)=\frac{\pi \cdot 2^{s}}{B(-s-n+1 / 2,-s+n+1 / 2)} .
$$


b) The bilinear form-valued function $s \mapsto B_{s}(f, g)$ is meromorphic on $\mathbb{C}$. The poles of this function are the points $s=0,{ }^{1} / 2,1,3 / 2, \ldots$

c) In the case $-1 / 2<s<1 / 2, s \neq 0$ the form $B_{s}$ is positively defined. In the case $2 s \in \mathbb{R} \backslash \mathbb{Z}$ the form $B_{s}$ is nondegenerate and its negative inertia index is finite.

d) Let us fix $2 s \in \mathbb{R} \backslash \mathbb{Z}$. Then the Stirling formula for $\Gamma$-function gives

$$
c_{n}(s)=C \cdot|n|^{2 s}\left(1+O\left(\frac{1}{n}\right)\right), \quad|n| \mapsto \infty
$$

Hence the form $B_{s}(\cdot, \cdot)$ is well-defined on the Sobolev space $W^{s}$.

Corollary. Let $2 s \notin \mathbb{Z}$. a) There exist the positive defined bilinear form $\tilde{B}_{s}$ on $W_{s}$ such that the form

$$
\psi=\tilde{B}_{s}-B_{s}
$$

has finite rank $\left(\psi\left(e^{i n \varphi}, e^{-i m \varphi}\right)=0\right.$ if $|n|$ of $|m|$ are sufficiently large $)$.

b) The scalar product $\tilde{B}_{s}$ is strongly equivalent to the scalar product (2.1).

Proof. a) see the property d),

b) see the formula (2.7).

\subsection{The Proof of Theorem 2.1 in the Case $s<0,2 s \notin \mathbb{Z}$}

We have to prove that $T_{s}^{*}(q) T_{s}(q)-E$ is a Hilbert-Schmidt operator in the scalar product $\tilde{B}_{s}(\cdot, \cdot)$,

$$
\tilde{B}_{s}\left(\left(T^{*}(q) T(q)-E\right) f, g\right)=\tilde{B}_{s}(T(q) f, T(q) g)-\tilde{B}_{s}(f, g) .
$$

Let $\tilde{B}_{s}=B_{s}+\psi$ [see (2.8)]. The rank of the form $\psi$ is finite. Hence the rank of $\psi(T(q) f, T(q) g)$ also is finite. So it is sufficient to prove that the operator $R(q)$ defined by the equality

$$
B_{s}(T(q) f, T(q) g)-B_{s}(f, g)=\langle R(q) f, g\rangle_{s}
$$

is a Hilbert-Schmidt operator.

$$
\begin{aligned}
& B_{s}(T(q) f, T(q) g)-B_{s}(f, g) \\
&=\int_{0}^{2 \pi} \int_{0}^{2 \pi}\left|\sin \left(\frac{\varphi-\psi}{2}\right)\right|^{-1-2 s} f(q(\varphi)) q^{\prime}(\varphi)^{1 / 2-s} g(q(\psi)) q^{\prime}(\psi)^{1 / 2-s} d \varphi d \psi \\
&-\int_{0}^{2 \pi} \int_{0}^{2 \pi}\left|\sin \left(\frac{\varphi-\psi}{2}\right)\right|^{-1-2 s} f(\varphi) g(\psi) d \varphi d \psi
\end{aligned}
$$

Let $p$ be the diffeomorphism inverse to $q$. We replace the variable in the first summand: $\theta=q(\varphi), \kappa=q(\psi)$. Then (2.9) equals

$$
\int_{0}^{2 \pi} \int_{0}^{2 \pi}\left[\frac{p^{\prime}(\theta)^{1 / 2+s} p^{\prime}(\kappa)^{1 / 2+s}}{\left|\sin \left(\frac{p(\theta)-p(\kappa)}{2}\right)\right|^{1+2 s}}-\frac{1}{\left|\sin \left(\frac{\theta-\kappa}{2}\right)\right|^{1+2 s}}\right] f(\theta) g(\kappa) d \theta d \kappa .
$$


Let us denote the expression in square brackets by $K(\theta, \kappa)$. Then

$$
K(\theta, \kappa)=\frac{1}{\left|\sin \left(\frac{\theta-\kappa}{2}\right)\right|^{2 s+1}} \lambda(\theta, \kappa)
$$

where

$$
\lambda(\theta, \kappa)=\left[\frac{p^{\prime}(\theta) p^{\prime}(\kappa) \sin ^{2}\left(\frac{\theta-\kappa}{2}\right)}{\sin ^{2}\left(\frac{p(\theta)-p(\kappa)}{2}\right)}\right]^{1 / 2+s}-1 .
$$

It is easy to see that $\lambda(\theta, \kappa)$ is the smooth function on the torus $S^{1} \times S^{1}, \lambda(\theta, \theta)=0$, $\lambda(\theta, \kappa)=\lambda(\kappa, \theta)$ and hence in a neighbourhood of the diagonal $\theta=\kappa$ we have

$$
\lambda(\theta, \kappa)=(\theta-\kappa)^{2} \mu(\theta, \kappa),
$$

where the function $\mu(\theta, \kappa)$ is smooth in a neighbourhood of the diagonal $\theta=\kappa$. Hence $\lambda(\theta, \kappa)$ can be represented in the form

$$
\lambda(\theta, \kappa)=\sin ^{2}\left(\frac{\theta-\kappa}{2}\right) \tau(\theta, \kappa),
$$

where $\tau(\theta, \kappa)$ is the smooth function on the torus $S^{1} \times S^{1}$. Thus

$$
K(\theta, \kappa)=\left|\sin \left(\frac{\theta-\kappa}{2}\right)\right|^{1-2 s} \tau(\theta, \kappa) .
$$

Lemma 2.4. Let $L\left(\varphi_{1}, \varphi_{2}\right)$ be a function of the torus.

$$
L\left(\varphi_{1}, \varphi_{2}\right)=\sum a_{m n} e^{\imath m \varphi_{1}} e^{-i n \varphi_{2}} .
$$

Then the operator $Q$ defined by equality

$$
\left\langle Q f_{1}, f_{2}\right\rangle_{s}=\int_{0}^{2 \pi} \int_{0}^{2 \pi} L\left(\varphi_{1}, \varphi_{2}\right) f_{1}\left(\varphi_{1}\right) f_{2}\left(\varphi_{2}\right) d \varphi_{1} d \varphi_{2}
$$

is a Hilbert-Schmidt operator in $W_{s}$ iff

$$
\sum_{n, m}\left(n^{2}+1\right)^{-s}\left(m^{2}+1\right)^{-s}\left|a_{m n}\right|^{2}<\infty .
$$

Proof. Consider the orthogonal (in the scalar product $\left.\langle\cdot, \cdot\rangle_{s}\right)$, basis $h_{n}(\varphi)=\left(h^{2}+\right.$ $1)^{-s / 2} e^{i n \varphi}$ in $W^{s}$. Then

$$
\left\langle R h_{m}, h_{n}\right\rangle=\left(n^{2}+1\right)^{-s / 2}\left(m^{2}+1\right)^{-s / 2} a_{m n} .
$$

Now let us represent the function $K(\theta, \kappa)$ in the form

$$
K(\theta, \kappa)=\sum_{j=0}^{k-1} L_{j}(\theta, \kappa)+\tilde{L}_{k}(\theta, \kappa)
$$


where

$$
\begin{aligned}
& L_{j}(\theta, \kappa)=\left|\sin \left(\frac{\theta-\kappa}{2}\right)\right|^{1-2 s+j} \mu_{j}(\theta), \\
& \tilde{L}_{k}(\theta, \kappa)=\left|\sin \left(\frac{\theta-\kappa}{2}\right)\right|^{1-2 s+k} \mu_{k}(\theta, \kappa),
\end{aligned}
$$

where functions $\mu_{1}(\theta), \ldots, \mu_{k-1}(\theta), \mu_{k}(\theta, \kappa)$ are smooth.

It is quite clear that for sufficiently large $k$ the function $\tilde{L}_{k}(\theta, \kappa)$ satisfies the condition (2.11).

Let us prove that

$$
L_{0}(\theta, \kappa)=\sum a_{m n} e^{\imath m \theta} e^{-i n \kappa}
$$

satisfies the condition (2.11) (the cases of $L_{1}, \ldots, L_{k-1}$ are analogous. Let us replace variables

$$
\psi_{1}=\theta_{1}, \quad \psi_{2}=-\theta+\kappa
$$

Then

$$
L_{0}\left(\psi_{1}, \psi_{2}\right)=\left|\sin \left(\psi_{2} / 2\right)\right|^{1-2 s} \mu_{0}\left(\psi_{1}\right)
$$

Let

$$
\begin{aligned}
\mu\left(\psi_{1}\right) & =\sum \alpha_{k} e^{i k \psi_{1}}, \\
\left|\sin \frac{\psi_{2}}{2}\right|^{1-2 s} & =\sum \beta_{\ell} e^{i \ell \psi_{2}} .
\end{aligned}
$$

Now we can rewrite $(2.11)$ in the form

$$
\sum\left((k+\ell)^{2}+1\right)^{-s}\left(\ell^{2}+1\right)^{-s}\left|\alpha_{k}\right|^{2}\left|\beta_{\ell}\right|^{2}<\infty
$$

It is easy to see that

$$
(k+\ell)^{2}+1 \leq 2\left(k^{2}+1\right)\left(\ell^{2}+1\right) .
$$

Hence the left side of (2.12) is less than

$$
2^{-s / 2}\left(\sum\left(\ell^{2}+1\right)^{-2 s}\left|\beta_{\ell}\right|^{2}\right)\left(\sum\left(k^{2}+1\right)^{-s}\left|\alpha_{k}\right|^{2}\right)
$$

But $\alpha_{k}$ rapidly decrease and hence the second multiplier is finite. Recall [see (2.7)] that

$$
\beta_{\ell}=C \cdot \ell^{2 s-2}(1+O(1 / \ell)), \quad|\ell| \rightarrow \infty,
$$

and hence the second multiplier is finite. 


\subsection{The Proof of Theorem 2.2 in the Case $s<0,2 s \notin \mathbb{Z}$}

Let us calculate

$$
\begin{aligned}
B_{s}\left(Q(A(\varphi)) F_{1}, Q(A(\varphi)) F_{2}\right)-B_{s}\left(F_{1}, F_{2}\right) \\
=\int_{0}^{2 \pi} \int_{0}^{2 \pi}\left|\sin \left(\frac{\varphi-\psi}{2}\right)\right|^{-2 s-1}\left\langle A(\varphi) F_{1}(\varphi), A(\psi) F_{2}(\psi)\right\rangle d \varphi d \psi \\
\quad-\int_{0}^{2 \pi} \int_{0}^{2 \pi}\left|\sin \left(\frac{\varphi-\psi}{2}\right)\right|^{-2 s-1}\left\langle F_{1}(\varphi), F_{2}(\psi)\right\rangle d \varphi d \psi,
\end{aligned}
$$

where $\langle\cdot, \cdot\rangle$ denote the usual scalar product in $\mathbb{R}^{n}$. The expression (2.13) equals

$$
\int_{0}^{2 \pi} \int_{0}^{2 \pi}\left[\frac{A^{t}(\psi) A(\varphi)-E}{\left|\sin \left(\frac{\varphi-\psi}{2}\right)\right|^{1+2 s}}\right] F_{1}(\varphi), F_{2}(\psi) d \varphi d \psi,
$$

where $A^{t}$ denote the transposed matrix.

Let $L(\varphi, \psi)$ be the term in the square brackets. Then

$$
L(\varphi, \psi)=\left|\sin \left(\frac{\varphi-\psi}{2}\right)\right|^{-2 s} \tau(\varphi, \psi),
$$

where $\tau(\varphi, \psi)$ is smooth operator-valued function. Now we can repeat the estimates from 2.6.

\subsection{Duality}

Let $f \in W^{s}, g \in W^{-s}$. Let us consider the pairing $\sigma: W^{s} \times W^{-s} \rightarrow \mathbb{R}$ given by the formula

$$
\sigma(f, g)=\int_{0}^{2 \pi} f g d \varphi .
$$

Let $f \in W^{s}$. Then the function

$$
\ell_{f}(g)=\sigma(f, g)
$$

is linear functional on $W^{-s}$. Following lemma is evident.

Lemma 2.5. The norm of the functional $\ell_{f}$ on $W^{-s}$ equals to the norm of the function $f$ in $W^{s}$. Thus the map $f \mapsto \ell_{f}$ is isometry of $W^{s}$ and the space dual to $W^{-s}$.

Furthermore the pairing $\sigma$ is Diff $^{\infty}$-invariant:

$$
\sigma\left(T_{s}(q) f, T_{-s}(q) g\right)=\sigma(f, g)
$$

for each $q \in \operatorname{Diff}^{\infty}$. Hence the dual operator to $T_{s}(q)$ is $T_{-s}(q)^{-1}$.

Thus the two following conditions are equivalent:

$$
T_{s}(q) \in G L O\left(W^{s}\right) \Leftrightarrow T_{-s}(q)^{-1} \in G L O\left(W^{-s}\right) .
$$


Now Theorem 2.1 is proved for all $s$ such that $2 s \notin \mathbb{Z}$.

Analogously the pairing $W_{n}^{s} \times W_{n}^{-s} \rightarrow \mathbb{R}$ given by

$$
\sigma_{n}\left(F_{1}, F_{2}\right)=\int_{0}^{2 \pi}\left\langle F_{1}(\varphi), F_{2}(\varphi)\right\rangle d \varphi
$$

is $\mathbb{L}^{\infty}(S O(n))$-invariant:

$$
\sigma_{n}\left(A(\varphi) F_{1}(\varphi), A(\varphi) F_{2}(\varphi)=\sigma_{n}\left(F_{1}, F_{2}\right) .\right.
$$

Thus Theorem 2.2 is proved for all $s$ such that $2 s \notin \mathbb{Z}$.

\subsection{The Proof of Theorem 2.1 in the Case $s \in \mathbb{N}$}

Let us calculate

$$
\begin{aligned}
B_{n}\left(T_{n}(q) f, T_{n}(q) g\right)-B_{n}(f, g) & \\
= & \int_{0}^{2 \pi}\left(f(q(\varphi)) q^{\prime}(\varphi)^{1 / 2-n}\right)^{(n)}\left(g(q(\varphi)) q^{\prime}(\varphi)^{1 / 2-n}\right)^{(n)} d \varphi \\
& \quad-\int_{0}^{2 \pi} f^{(n)}(\varphi) g^{(n)}(\varphi) d \varphi+\ldots
\end{aligned}
$$

(other summands have finite rank and we don't write them)

$$
\begin{aligned}
= & \int_{0}^{2 \pi} f^{(n)}(q(\varphi)) q^{\prime}(\varphi)^{1 / 2} g^{(n)}(q(\varphi)) q^{\prime}(\varphi)^{1 / 2} d \varphi \\
& +\sum_{0 \leq i \leq n, 0 \leq j \leq n, i+\jmath>0} \int_{0}^{2 \pi} R_{\imath \jmath}(\varphi) f^{(n-\imath)}(q(\varphi)) g^{n-j)}(q(\varphi)) d \varphi \\
& -\int_{0}^{2 \pi} f^{(n)}(\varphi) g^{(n)}(\varphi) d \varphi+\ldots=
\end{aligned}
$$

(where $R_{i j}$ are some smooth functions)

$$
=\sum_{0 \leq \imath \leq n, 0 \leq \jmath \leq n, i+j>0} \int_{0}^{2 \pi} R_{\imath j}(\varphi) f^{(n-\imath)}(q(\varphi)) g^{(n-\jmath)}(q(\varphi)) d \varphi+\ldots
$$

Let us consider the form

$$
\int_{0}^{2 \pi} R_{\imath j}(\varphi) f^{(n-i)}(q(\varphi)) g^{(n-j)}(q(\varphi)) d \varphi=\int_{0}^{2 \pi} \tilde{R}_{i j}(\varphi) f^{(n-\imath)}(\varphi) g^{(n-j)}(\varphi) d \varphi
$$


where $R_{i j}(\varphi)=\tilde{R}_{\imath j}(q(\varphi)) q^{\prime}(\varphi)^{-1}$. This form is the continuous map $W^{n-i} \times$ $W^{n-j} \rightarrow \mathbb{R}$. Hence the operator $\mathscr{D}_{i j}$ defined by the equality

$$
B_{n}\left(\mathscr{D}_{i j} f, g\right)=\int_{0}^{2 \pi} \tilde{R}_{\imath \jmath}(\varphi) f^{(n-i)}(\varphi) g^{(n-j)}(\varphi) d \varphi
$$

is the pseudodifferential operator of the order $-(i+j)$. But $i+j \geq 1$, and thus $\mathscr{D}_{i j}$ is Hilbert-Schmidt operator (see [T]).

Remark. In the case $n=1$ (the Wiener measure) the following nice formula containing Schwartz derivative is obtained:

$$
B_{1}\left(T_{1}(q) f, T_{1}(q) g\right)-B_{1}(f, g)=\int_{0}^{2 \pi} f(p(\varphi)) g(p(\varphi))\left[\frac{1}{2} \frac{p^{\prime \prime \prime}}{p^{\prime}}-\frac{3}{4} \frac{\left(p^{\prime \prime}\right)^{2}}{\left(p^{\prime}\right)^{3}}\right] d \varphi+\ldots
$$

2.10. The Proof of Theorem 2.2 in the Case $s=n \in \mathbb{N}$

Let us calculate

$$
\begin{aligned}
& B_{n}\left(A(\varphi) F_{1}(\varphi), A(\varphi) F_{2}(\varphi)\right)-B_{n}\left(F_{1}(\varphi), F_{2}(\varphi)\right) \\
& =\int_{0}^{2 \pi}\left\langle\left(A(\varphi) F_{1}(\varphi)\right)^{(n)},\left(A(\varphi) F_{2}(\varphi)\right)^{(n)}\right\rangle d \varphi-\int_{0}^{2 \pi}\left\langle F_{1}^{(n)}(\varphi), F_{2}^{(n)}(\varphi)\right\rangle d \varphi+\ldots \\
& \quad(\text { we omit terms of finite rank) } \\
& =\int_{0}^{2 \pi}\left\langle A(\varphi) F_{1}^{(n)}(\varphi), A(\varphi) F_{2}^{(n)}(\varphi)\right\rangle d \varphi \\
& \quad+\sum_{\imath \geq 0, j \geq 0, i+j>0} \int_{0}^{2 \pi} N_{i j}(\varphi) F_{1}^{(n-1)}(\varphi) F_{2}^{(n-j)}(\varphi) d \varphi-\int_{0}^{2 \pi} F_{1}^{(n)}(\varphi) F_{2}^{(n)}(\varphi) d \varphi
\end{aligned}
$$

The first summand and the last summand are equal. For other summands valid arguments are in 2.9 .

\subsection{We Omit the Proof in the Case $s=k+1 / 2$}

\section{Decompositions of Dynamical Systems (the Case of the Group Diff ${ }^{\infty}$ )}

In Sect. 2 we constructed a series of embeddings Diff ${ }^{\infty}$ to the groups $G L O\left(W^{s} \mid W^{s-1 / 2-\varepsilon}\right)$ and hence the series of quasi-invariant actions of Diff ${ }^{\infty}$ on the spaces with Gauss measures. Recall that the group Diff ${ }^{\infty}$ acts on the space $W^{s-1 / 2-\varepsilon}$ by the formula

$$
T_{s}(q) f(\varphi)=f(q(\varphi)) q^{\prime}(\varphi)^{1 / 2-s}
$$

and $T_{s}(q) \in G L O\left(W^{s} \mid W^{s-1 / 2-\varepsilon}\right)$. We want to discuss ergodic properties of these actions for different $s \geq 0$. 
$\alpha$. The Case $s=0$

\subsection{This Case is Not Interesting}

The space $W^{0}$ is the usual $L^{2}\left(S^{1}\right)$. The group Diff ${ }^{\infty}$ acts in $L^{2}\left(S^{1}\right)$ by orthogonal operators

$$
T_{0}(q) f(\varphi)=f(q(\varphi)) q^{\prime}(\varphi)^{1 / 2}
$$

and thus we have the embedding Diff ${ }^{\infty} \rightarrow O\left(W^{0}\right) \subset G L O\left(W^{0}\right)$.

The representation of Diff ${ }^{\infty}$ in $L^{2}\left(\hat{W}^{0}\right)$ is the direct sum of symmetric powers of representation $T_{0}(q)$ (see 1.5 ).

B. The Case $0<s<1 / 2$

\subsection{The Scalar Product}

Let us consider the following scalar product

$$
B_{s}(f, g)=\int_{0}^{2 \pi} \int_{0}^{2 \pi}\left|\sin \left(\frac{\varphi-\psi}{2}\right)\right|^{-1-s} f(\varphi) g(\psi) d \varphi d \psi
$$

in $W^{s}$. We have seen that this scalar product is strongly equivalent to the scalar product (2.1).

The group Diff ${ }^{\infty}$ acts in $W^{s}$ by the formula

$$
T_{s}(q) f(\varphi)=f(q(\varphi)) q^{\prime}(\varphi)^{1 / 2-s} .
$$

Now let us consider Möbius transformations of the disk $|z| \leq 1$ on the complex plane:

$$
M\left(\begin{array}{cc}
\alpha & \beta \\
\bar{\beta} & \bar{\alpha}
\end{array}\right): z \mapsto \frac{\alpha z+\beta}{\bar{\beta} z+\bar{\alpha}}
$$

where $|\alpha|^{2}-|\beta|^{2}=1$. The group of all Möbius transormations is isomorphic to the group $P S L_{2}(\mathbb{R})$. Möbius transformations map the circle $|z|=1$ to itself. Hence we obtained the canonical embedding

$$
\mathrm{PSL}_{2}(\mathbb{R}) \rightarrow \operatorname{Diff}^{\infty}
$$

and we can identiy $P S L_{2}(\mathbb{R})$ with the subgroup in Diff ${ }^{\infty}$.

Proposition 3.1. Let $q \in P S L_{2}(\mathbb{R})$. Then the operator $T_{s}(q)$ is orthogonal.

This proposition is a well-known fact (Bargmann (1948), see [GGV]). The representations $T_{s}$ of the group $P S L_{2}(\mathbb{R})$ are called "representations of complementary series" $(0<s<1)$.

Remark. Of course all strongly equivalent scalar products in $W^{s}$ are equivalent for our purposes. But in the case $0<s<1$ the scalar product (3.2) is the best (it is the only $P S L_{2}(\mathbb{R})$-invariant scalar product in $W^{s}$ ).

In particular transformations from $P S L_{2}(\mathbb{R})$ preserve the measure on $\hat{W}_{s}$. 


\subsection{Ergodicity}

Proposition 3.2. Let $0<s<1$. Then the action $T_{s}$ of $P S L_{2}(\mathbb{R})$ on the space $\hat{W}_{s}$ is ergodic.

Proof. We have to show that there exist unique $P S L_{2}(\mathbb{R})$-invariant vectors in $L^{2}\left(\hat{W}^{s}\right)$. The representation of $P S L_{2}(\mathbb{R})$ in $L^{2}\left(\hat{W}_{s}\right)$ is the direct sum of the symmetric powers $S^{k} T_{s}$ of representation $T_{s}$ (see Sect. 1.5). The symmetric power $S^{0} T_{s}$ is the onedimensional representation. Decompositions of tensor products of representations of $P S L_{2}(\mathbb{R})$ are known (see $[\mathrm{Re}]$ ), and it is known that a one-dimensional representation cannot appear in decompositions of tensor products of representations of $P S L_{2}(\mathbb{R})$. Thus there are no $P S L_{2}(\mathbb{R})$-invariant vectors in the symmetric powers $S^{k} T_{s}$ for $k>0$.

\section{$\gamma$. The Case $s=1 / 2$ (Highest Weight Case)}

\subsection{The Scalar Product}

The case $s=1 / 2$ is exceptional. In this case the representation $T_{s}=T_{1 / 2}$

$$
T_{1 / 2}(q) f(\varphi)=f(q(\varphi))
$$

of the group Diff ${ }^{\infty}$ is reducible. It is evident that the subspace $\mathbb{R} \cdot 1$ of all constants as Diff ${ }^{\infty}$-invariant. By this reason it is more natural to consider the action of Diff ${ }^{\infty}$ in the factor-space $\tilde{W}^{1 / 2}=W^{1 / 2} / \mathbb{R}$.

Let us provide $\tilde{W}^{1 / 2}$ by the scalar product

$$
\langle f, g\rangle=\int_{0}^{2 \pi} \int_{0}^{2 \pi} \operatorname{ctg}\left(\frac{\varphi-\psi}{2}\right) f(\varphi) g^{\prime}(\psi) d \varphi d \psi
$$

The usual considerations show that $T_{1}(q) \in G L O\left(\tilde{W}^{1}\right)$ (see $[\mathrm{N} 2, \mathrm{~N} 6]$ ).

Proposition 3.3. The action of $P S L_{2}(\mathbb{R})$ in $\hat{\tilde{W}}^{1 / 2}$ is ergodic.

Proof. Let $q \in P S L_{2}(\mathbb{R})$. The operators $T_{1 / 2}(q)$ are orthogonal in the scalar product (3.3). Now we can repeat the proof of Proposition 3.2.

Remark. This action allows us to construct highest weight representations of Diff ${ }^{\infty}$, see Sect. 6.1.

\section{$\delta$. The Case $s=1$ (The Wiener Measure)}

\subsection{Invariant Subsets}

Let us consider the following subsets in the space of all Wiener trajectories:

$\Omega_{+}-$the set of positive functions [i.e. $f(\varphi)>0$ for each $\varphi$ ]

$\Omega_{-}-$the set of negative functions.

$\Omega_{0}-$ the set of functions which have zeroes.

It is evident that subsets $\Omega_{+}, \Omega_{-}, \Omega_{0}$ are Diff ${ }^{\infty}$-invariant and it is evident that they have nonzero measures. 


\subsection{Integrals}

Let $f \in \Omega_{+}$. Let us consider the following functional on $\Omega_{+}$:

$$
I(f)=\int_{0}^{2 \pi} \frac{d \varphi}{f^{2}(\varphi)} .
$$

Proposition 3.4. $I\left(T_{1}(q) f\right)=I(f)$.

Proof. Evident.

Thus the functional $I(f)$ is Diff ${ }^{\infty}$-invariant and the subsets

$$
\Omega_{+}^{\alpha, \beta}=\left\{f \in \Omega_{+}: \alpha \leq I(f) \leq \beta\right\}
$$

also are invariant.

Hypothesis. a) The conditional measures $\mu_{C}$ on the "surfaces" $I(f)=C$ exist for all $C>0$.

b) The measures $\mu_{C}$ are Diff $^{\infty}$-quasi-invariant.

\subsection{Imprimitivity Systems}

Let a group $G$ act on a set $M$. Let $M=\bigcup_{a \in A} M_{a}$ be a partition of $M$ (i.e. $M_{\alpha} \cap M_{\beta}=\emptyset$ if $\alpha \neq \beta$ ). The partition $\left\{M_{\alpha}\right\}$ is called an imprimitivity system if for arbitrary $g \in G$ and $a \in A$ the set $g M_{a}^{\alpha}$ is one of the subsets $M_{b}$. Let $M=\bigcup_{a \in A} M_{a}$ be the imprimitivity system. Then we have the action of the group $G$ on the factor set $A$ (by definition $g a=b$ if $g M_{a}=M_{b}$ ).

Now let the set $M$ be the space with probabilitic measure $\mu$. Let $M=\bigcup_{a \in A} M_{a}$ be a partition. We define the measure $\alpha$ on the factorspace $A$. By definition a subset $E \subset A$ is measurable if the subset $\bigcup_{a \in E} M_{a}$ is measurable in $M$. Let $E \subset A$ be the measurable subset. Then $\alpha(E)=\mu\left(\bigcup_{a \in E} M_{a}\right)$.

Remark. For some partitions $M=\bigcup_{a \in A} M_{a}$ the measure $\alpha$ on $A$ has pathological properties. In fact it is necessary to control measurability of the partition (see [Ro]). Lower measurability of partitions in all cases is quite evident.

Proposition 3.5. Let us consider quasi-invariant action of the group $G$ on space $M$ with a measure $\mu$. Let $M=\bigcup_{a \in A} M_{a}$ be an imprimitivity system. Then the canonical measure $\alpha$ on $A$ is G-quasi-invariant.

Proof. Let $E \subset A, \alpha(E) \neq 0$. We have to prove that $\alpha(g E) \neq 0$. Indeed

$$
\begin{aligned}
\alpha(g E) & =\mu\left(\bigcup_{b \in g E} M_{b}\right)=\mu\left(\bigcup_{a \in E} M_{g a}\right) \\
& =\mu\left(\bigcup_{a \in E} g M_{a}\right)=\mu\left(g\left(\bigcup_{a \in E} M_{a}\right)\right) .
\end{aligned}
$$


But $\mu\left(\bigcup_{a \in E} M_{a}\right)=\alpha(E) \neq 0$, and hence (by a quasi-invariance)

$$
\mu\left(g\left(\bigcup_{a \in E} M_{a}\right)\right) \neq 0
$$

\subsection{Projectivization of $\Omega_{+}$}

The following lemma is evident.

Lemma 3.1. Let us define for each $f \in \Omega_{+}$the subset $M^{f}=\left\{g \in \Omega_{+}: g / f=\right.$ const $\}$. Then the system of subsets $M^{f}$ is an imprimitivity system.

Let $S$ be the set of all substs $M^{f}$ (in other words $S$ is the space of positive functions defined up to a multiplier). Let $\sigma$ be the projection of the Wiener measure on $\Omega_{+}$to $S$. By Proposition 3.5 the measure $\sigma$ on $S$ is Diff ${ }^{\infty}$-quasi-invariant.

Let Diff ${ }^{1}$ be the group of $C^{1}$-diffeomorphisms of the circle preserving orientation and let $\mathbb{T}$ be the group of rotations of the circle.

Let $p$ be an element of the homogeneous space $\mathbb{T} \backslash$ Diff $^{1}$, this means that $p$ is a diffeomorphism defined up to equivalence $p \sim r_{\theta} \circ p$, where $r_{\theta} \in \mathbb{T}$ is a rotation of the circle. Let us define a map $R: \mathbb{T} \backslash \operatorname{Diff}^{(1)} \rightarrow S$ given by

$$
R(p)=p^{\prime}(\varphi)^{-1 / 2}
$$

Lemma 3.2. The map $R$ is bijection.

Proof. Let $f \in S$. We can think that $f$ is a fucntion normed by the conditon $I(f)=1$, see (3.4). Let

$$
Q(f)=\int_{0}^{\varphi} \frac{d \varphi}{f^{2}(\varphi)}
$$

Then the map $Q$ is inverse to the map $R$.

We se that it is possible to identify the space $S$ and the space $\mathbb{T} \backslash \operatorname{Diff}^{(1)}$, and thus we constructed a Diff ${ }^{\infty}$-quasi-invariant measure on the homogeneous space $\mathbb{T} \backslash \operatorname{Diff}^{(1)}$.

\subsection{The Measure on the Space of Cantor Subsets of the Circle}

It is well-known that the set of zeroes of the "general" Wiener path $f(\varphi) \in \Omega_{0}$ is a nowhere dense closed subset (see [L]).

Let $\mathscr{R}$ be the space of closed subsets of the circle (provided by natural borel structure [I]). Let us consider the projection $\omega: \Omega_{0} \rightarrow \mathscr{R}$ defined by the following rule: the set $\pi(f)$ is the set of zeroes of a function $f$. It is evident that the map $\pi$ commutes with the action of the group Diff ${ }^{\infty}$. Let the measure $\varrho$ on $\mathscr{B}$ be the image of the Wiener measure on $\Omega_{0}$. Then by Proposition 3.5 the measure $\varrho$ on $\mathscr{R}$ is quasi-invariant. 
ع. The Case $1<s<2$

\subsection{Let $s>1$}

Then the space $\hat{W}^{s}$ consists of continuous functions and we can repeat all considerations of Sects. 3.5-3.8 (and Sect. 3.9 if $s<3 / 2$ ).

The invariant integral on $\Omega_{+}$in this case is given by the formula

$$
I_{s}(f)=\int_{0}^{2 \pi} \frac{d \varphi}{f(\varphi)^{2 /(2 s-1)}}
$$

\section{$\zeta$. The Case $s>2$}

\subsection{Let $t>3 / 2$}

Then elements of the space $W^{t}$ are $C^{1}$-smooth functions. Thus the space $\hat{W}^{s} \cong$ $W^{s-1 / 2-\varepsilon}$ in this case consists of functions with continuous derivatives.

Again we have three invariant subsets: the set $\Omega_{+}$of positive functions, the set $\Omega_{-}$of negative functions and the set $\Omega_{0}$ of functions which have zero.

For the case $\Omega_{+}$it is possible to repeat all considerations of Sects. 3.5, 3.8.

\subsection{Set $\Omega_{0}$}

"General" function $f \in \Omega_{0}$ has a finite even number of zeroes $t_{1}, \ldots, t_{2 p}$ and $f^{\prime}\left(t_{j}\right) \neq 0$.

Let $\Omega_{0}^{2 p}$ be the set of functions $f \in \Omega_{0}$ which have $2 p$ zeroes. It is evident that the sets $\Omega_{0}^{2 p}$ are invariant, it is easy to show that measure of $\Omega_{0}^{2 p}$ is not zero.

For each $f \in \Omega_{0}^{2 p}$ we construct the following collections of numbers

$$
I_{s}^{1}(f)=\int_{t_{1}}^{t_{2}}|f(\varphi)|^{-\frac{2}{2 s-1}} d \varphi, \ldots, I_{s}^{2 p}(f)=\int_{t_{2 p}}^{t_{1}}|f(\varphi)|^{-\frac{2}{2 s-1}} d \varphi .
$$

It is evident that the collection

$$
I_{s}^{1}(f), I_{s}^{2}(f), \ldots, I_{s}^{2 p}(f)
$$

defined up to cyclic permutations of type

$$
\left(\begin{array}{cccc}
1 & 2 & 3 & 4 \\
2 m & 2 m+1 & 2 m+2 & 2 m+3
\end{array} \cdots\right)
$$

is invariant of the function $f$. 


\section{Decomposition of Dynamical Systems (Loop Groups)}

\subsection{Invariants}

Let us consider the action of the group $\mathbb{L}^{\infty}(S O(m))$ in $\hat{W}_{m}^{s}$ constructed in Sect. 2.3. Let $s>1 / 2$. Then the canonical extension $\hat{W}_{n}^{s}$ of $W_{n}^{s}$ can be looked upon as the subspace in $L^{2}\left(S^{1}, \mathbb{R}^{n}\right)$.

The following statement is evident.

Proposition 4.1. Let $s>1 / 2$. For each $\operatorname{arc}(\alpha, \beta) \subset S^{1}$ the functional

$$
J_{\alpha, \beta}(f)=\int_{\alpha}^{\beta}\langle f, f\rangle d \varphi
$$

is $\mathbb{L}^{\infty}(S O(m))$-invariant.

Let $s \geq 1$. Then $\hat{W}_{m}^{s}$ can be looked upon as some space of continuous functions (for $s=1$ we have Wiener measure, for $s>1$ elements of the space $W_{m}^{2-1 / 2-\varepsilon}$ are continuous functions (for small $\varepsilon>0$ )). It is evident that for each $\varphi_{0} \in S^{1}$ the functional

$$
J_{\varphi_{0}}(f)=\left\langle f\left(\varphi_{0}\right), f\left(\varphi_{0}\right)\right\rangle
$$

is $\mathbb{L}^{\infty}(S O(m))$-invariant.

\subsection{Remark Random Walking on Random Sphere}

Let $s \geq 1$. Here we again have the question of conditional measures:

a) Let $r(\varphi)$ be a nonnegative continuous function. For which $r(\varphi)$ does there exist a conditional measure on the set of functions $f \in \hat{W}_{m}^{s}$ satisfying

$$
J_{\varphi_{0}}(f)=r\left(\varphi_{0}\right)
$$

(for each $\varphi_{0}$ )?

b) Is this measure $\mathbb{L}^{\infty}(S O(m))$-quasi-invariant?

Remark. I don't know the answers to these questions. Of course conditional measures exist almost everywhere, but there are no general theorems which provide quasiinvariance.

Remark. In the case $s=1$ it is possible to interpret such measures as random walking on spheres of random radius.

\subsection{Non-Wiener Walkings on the Sphere}

Let $s \geq 1$ (and $m>1$ ). Let $\Omega \subset \hat{W}_{m}^{s}$ be the set of paths $f \in \hat{W}_{m}^{s}$ which don't pass zero. It is simple to show that the measure of $\hat{W}_{m}^{s} \backslash \Omega$ is zero (for our purposes it is sufficient to know that the measure of $\Omega$ is not zero).

Let $S^{m-1}$ be an $(n-1)$-dimensional sphere. Let $\mathbb{L}^{0}\left(S^{m-1}\right)$ be the set of continuous functions $S^{1} \rightarrow S^{m-1}$. The group $\mathbb{L}^{\infty}(S O(m))$ acts on $\mathbb{L}^{0}\left(S^{m-1}\right)$ by evident way. 
Let us consider the equivariant map $\pi$ from $\Omega \subset \hat{W}_{m}^{s}$ to $\mathbb{L}^{0}\left(S^{m-1}\right)$ defined by formula

$$
\pi f(\varphi)=\frac{f(\varphi)}{r(\varphi)}
$$

where $r(\varphi)=\sqrt{\langle f(\varphi), f(\varphi)\rangle}$.

Let $\nu_{s}$ be the projection of the canonical measure in $\hat{W}_{n}^{s}$. By Proposition 3.5. The measure $\nu_{s}$ on $\mathbb{L}^{0}\left(S^{m-1}\right)$ is $\mathbb{L}^{\infty}(S O(m))$-quasi-invariant.

Remark. The measure $\nu_{1}$ is not a Wiener measure on paths on the sphere.

\subsection{Diff ${ }^{\infty}$-quasi-invariance of the Measures $\nu_{s}$}

The group Diff ${ }^{\infty}$ acts on $\mathbb{L}^{0}\left(S^{m-1}\right)$ by replacing the variable:

$$
g(\varphi) \mapsto g(q(\varphi))
$$

where $q(\varphi) \in \operatorname{Diff}^{\infty}$.

Proposition 4.1. The measures $\nu_{s}$ are Diff $^{\infty}$-quasi-invariant.

Proof. Let Diff ${ }^{\infty}$ act in $W_{m}^{s}$ by the transformations

$$
T_{s}(q) f(\varphi)=q^{\prime}(\varphi) f(\varphi)^{1 / 2-s} .
$$

It is easy to see that $T_{s}(q) \in G L O\left(W_{s}^{m}\right)$ (it is an evident variation of Theorem 2.1). Thus the group Diff ${ }^{\infty}$ acts on the space $\hat{W}_{m}^{s}$. It is evident that the map $\pi$ given by the formula (4.1) is Diff $^{\infty}$-equivariant. Now we can apply Proposition 3.5.

\section{Comments (Dynamical Systems)}

\subsection{Quasi-Invariant Measures on Diff ${ }^{1}$}

Let Diff ${ }^{1}$ be the group of $C^{1}$-smooth diffeomorphisms of the circle. In Sect. 3 we constructed some series of Diff ${ }^{\infty}$-quasi-invariant measures on $\mathbb{T} \backslash$ Diff $^{1}$ depending on parameter $s \geq 1$. Let us denote these measures by $\mu_{s}$.

Let us consider the map

$$
\lambda: \operatorname{Diff}^{1} \rightarrow S^{1} \times\left(\mathbb{T} \backslash \operatorname{Diff}^{1}\right)
$$

defined by the formula

$$
\lambda(p)=p(0) \times \tilde{p},
$$

where $\tilde{p} \in \mathbb{T} \backslash$ Diff $^{1}$ is the projection of $p \in \operatorname{Diff}^{1}$. It is easy to see that $\lambda$ is a topological isomorphism.

Now let us provide the space $S^{1} \times\left(\mathbb{T} \backslash D\right.$ iff $\left.^{1}\right)$ by the product $\tilde{\mu}_{s}$ of the Lebesgue measure $d \varphi$ on $S^{1}$ and the measure $\mu_{s}$ on $\mathbb{T} \backslash$ Diff $^{1}$. The measure $\tilde{\mu}_{s}$ is Diff ${ }^{\infty}$-quasiinvariant. But

$$
s^{1} \times\left(\mathbb{T} \backslash \operatorname{Diff}^{1}\right) \cong \operatorname{Diff}^{1}
$$

and thus we can consider $\tilde{\mu}_{s}$ as a Diff ${ }^{\infty}$-right-quasi-invariant measure on Diff ${ }^{1}$. 


\subsection{Quasi-Invariant Measures on Loop Groups}

Let $s>1$. Our purpose is to construct $\mathbb{L}^{\infty}(S O(n))$-quasi-invariant measures on the group $\mathbb{L}^{0}(S O(n))$ of continuous functions $S^{1} \rightarrow S O(n)$.

Let us denote by $\mathbb{L}_{0}^{0}(S O(n))$ the group of continuous functions $g: S^{1} \rightarrow S O(n)$ satisfying the condition $g(0)=E$. Let $\mathbb{L}_{0}^{\infty}(S O(n))=L^{\infty}(S O(n)) \cap L_{0}^{0}(S O(n))$.

Step 1. The canonical embedding $\mathbb{L}^{\infty}(S O(n)) \rightarrow \mathbb{L}^{\infty}(S O(n(n+1) / 2))$. Let us denote by $M_{n}$ the space of real symmetric $n \times n$ matrices.

The space $M_{n}$ is a $n(n+1) / 2$ )-dimensional real Euclidean space provided by the scalar product

$$
\langle X, Y\rangle=\operatorname{tr}(X Y) .
$$

Thus we can identify the spaces $M_{n}$ and $\mathbb{R}^{N}$, where $N=n(n+1) / 2$.

The group $S O(n)$ acts in $M_{n} \cong \mathbb{R}^{N}$ by the transformations

$$
\kappa(g): X \mapsto g^{t} X g .
$$

It is evident that such transformations are orthogonal, i.e. $\kappa(g) \in S O(N)$. Hence the group $\mathbb{L}_{0}^{\infty}(S O(n))$ acts in $W_{N}^{s}$ by the transformations

$$
\kappa(g(\varphi)): X(\varphi) \mapsto g^{t}(\varphi) X(\varphi) g(\varphi) .
$$

We have $\kappa(g(\varphi)) \in \mathbb{L}_{0}^{\infty}(S O(N))$, and hence

$$
\kappa(g(\varphi)) \in G L O\left(W_{N}^{s} \mid W_{N}^{s-1 / 2-\varepsilon}\right) .
$$

Let $v \in M_{n} \cong \mathbb{R}^{N}$. Let us denote by $W_{N}^{t}[v]$ the set of functions $f \in W_{N}^{t}[v]$ satisfying the condition $f(0)=v$. It is easy to see that the sets $W_{N}^{s-1 / 2-\varepsilon}[v]$ are $\mathbb{L}_{0}^{\infty}(S O(n))$-invariant.

Step 2. The construction of the conditional measures on $W_{N}^{s-1 / 2-\varepsilon}[v]$. Let us notice that $W_{N}^{s-1 / 2-\varepsilon}[0]$ is linear space and $\widehat{W_{N}^{s}(0)} \cong W_{N}^{s-1 / 2-\varepsilon}[0]$. It is quite evident that

$$
\kappa(g(\varphi)) \in G L O\left(W_{N}^{s}[0] \mid W_{N}^{s-1 / 2-\varepsilon}[0]\right) .
$$

Let $f(\varphi) \in W_{N}^{t}[0]$. Then $v+f(\varphi) \in W_{N}^{t}[v]$, and hence we can identify the spaces $W_{N}^{t}[0]$ and $W_{N}^{t}[v]$. The action $(5.1)$ of $\mathbb{L}_{0}^{\infty}(S O(n))$ in $W^{s-1 / 2-\varepsilon}[v]$ corresponds to the affine action

$$
g(\varphi): X(\varphi) \mapsto g^{t}(\varphi) X g(\varphi)+g^{t}(\varphi) v g(\varphi)-v
$$

in $W_{N}^{s-1 / 2-\varepsilon}[0] \cong \hat{W}_{N}^{s}[0]$. The functions $g^{t}(\varphi) v g(\varphi)-v$ are smooth, hence

$$
g^{t}(\varphi) v g(\varphi)-\varphi \in W_{N}^{s}[0] \text {. }
$$

Hence (see Sect. 1.3) the canonical measure in $W_{N}^{s-1 / 2-\varepsilon}[0] \cong \hat{W}_{N}^{s}[0]$ is quasiinvariant with respect to transformations (5.2). But we have identified $W_{N}^{s-1 / 2-\varepsilon}$ [0] and $W_{N}^{s-1 / 2-\varepsilon}[v]$ and thus we obtained $\mathbb{L}_{0}^{\infty}(S O(n))$-quasi-invariant measure on the set $W_{N}^{s-1 / 2-\varepsilon}[v]$.

Step 3. Imprimitivity system on $W_{N}^{s-1 / 2-\varepsilon}[v]$. Let $L \subset M_{n}$ be the set of matrices with pairly different eigenvalues. Let $v \in L$. It is easy to see that the manifold $M_{n} \backslash L$ has codimension 2. Hence the "general path" $f(\varphi) \in W_{N}^{s-1 / 2-\varepsilon}[v]$ doesn't 
intersect $M_{n} \backslash L$ (we omit the simple proof of this fact). Let $\Omega^{(v)}$ be the set of paths $f(\varphi) \in W^{s-1 / 2-\varepsilon}[v]$ which don't intersect $M_{n} \backslash L$.

Let us denote by $\Delta_{n}$ the set of all collections $\left(\ell_{1}, \ldots, \ell_{n}\right)$ of pairly orthogonal lines in $\mathbb{R}^{n}$. The group $S O(n)$ acts on $\Delta_{n}$ by the evident way. It is clear that the space $\Delta_{n}$ is $S O(n)$-homogeneous and the stabilizer of a point is some finite subgroup $\Gamma_{n}$ $\left(\Gamma_{n}\right.$ is the semidirect product of the symmetric group $S_{n}$ and Abel group $(\mathbb{Z} / 2 \mathbb{Z})^{n-1}$, i.e. $\left.\Gamma_{n}=S_{n} \ltimes(\mathbb{Z} / 2 \mathbb{Z})^{n-1}\right)$.

Define the canonical $S O(n)$-equivariant map $\pi: L \rightarrow \Delta_{n}$. Let $X \in L$, then $\pi(X)$ is the set of eigenlines of $X$.

Denote by $\mathbb{L}^{0}\left(\Delta_{n}\right)_{v}$ the set of continuous functions $g: S^{1} \rightarrow \Delta_{n}$ satisfying the condition $g(0)=v$. Let $f \in \Omega^{v}$. Then $\pi(f(\varphi)) \in \mathbb{L}^{0}\left(\Delta_{n}\right)_{\pi(v)}$. So we constructed $\mathbb{L}_{0}^{\infty}\left(S O(n)\right.$ )-equivariant map $\Omega^{v} \rightarrow \mathbb{L}\left(\Delta_{n}\right)_{\pi(v)}$, and hence (by Proposition 3.5) we obtained $\mathbb{L}_{0}^{\infty}(S O(n))$-quasi-invariant measure on $\mathbb{L}^{0}\left(\Delta_{n}\right)_{\pi(v)}$.

Step 4. Lifting to $\mathbb{L}^{0}(S O(n))$. The space $\Delta_{n}$ is not simply connected, the fundamental group of $\Delta_{n}$ is the finite group $\Gamma_{n}$. Let us denote by $\mathbb{L}^{0}\left(\Delta_{n}\right)_{\pi(v)}^{e}$ the set of paths $p(\varphi) \in \mathbb{L}^{0}\left(\Delta_{n}\right)_{\pi(v)}^{e}$ homotopical to constant path. It is easy to show that the measure of $\mathbb{L}^{0}\left(\Delta_{n}\right)_{\pi(v)}^{e}$ is not zero.

Let $\lambda: S O(n) \rightarrow \Delta_{n}$ be the canonical $S O(n)$-equivariant projection such that $\lambda(E)=\pi(v)$. This projection identifies the spaces $\mathbb{L}_{0}^{0}(S O(n))$ and $\mathbb{L}^{0}\left(\Delta_{n}\right)_{\pi(v)}^{e}$ and hence we obtained left $\mathbb{L}_{0}^{\infty}(S O(n))$-quasi-invariant measure on $\mathbb{L}_{0}^{0}(S O(n))$.

Step 5 . We identify the spaces $\mathbb{L}^{0}(S O(n))$ and $S O(n) \times \mathbb{L}_{0}^{0}(S O(n))$ by the following way:

$$
g(\varphi) \rightarrow\left(f(0), g^{-1}(0) g(\varphi)\right) \in S O(n) \times \mathbb{L}^{0}(S O(n)) .
$$

Consider the product $\mu_{s}$ of the Haar measure on $S O(n)$ and the measure on $\mathbb{L}^{0}(S O(n))$ constructed above.

Lemma 5.1. The measure $\mu_{s}$ is left $\mathbb{L}^{\infty}(S O(n))$-quasi-invariant.

Proof. Let $h(t) \in \mathbb{L}_{0}^{\infty}(S O(n)), b \in S O(n), g(t) \in \mathbb{L}_{0}^{0}(S O(n))$. Then

$$
h(t) b g(t)=b\left[b^{-1} h(t) b\right] g(t),
$$

and for each fixed $b \in S O(n)$ the conditional measure on the fibre $q(0)=b$ in $\mathbb{L}^{0}(S O(n))$ is $\mathbb{L}_{0}^{\infty}(S O(n))$-quasi-invariant. Hence $\mu_{s}$ is $\mathbb{L}_{0}^{\infty}(S O(n))$-quasi-invariant. The quasi-invariance with respect to $S O(n) \subset \mathbb{L}^{\infty}(S O(n))$ is evident.

Remark. In fact the support of the measure $\mu_{s}$ is contained in the group $\mathbb{L}^{[s-1 / 2]}(S O(n))$ of all loops of the class $C^{[s-1 / 2]}$.

\subsection{Diff ${ }^{\infty}$-Quasi-Invariance}

Let us denote by Diff ${ }_{0}^{\infty}$ the group of smooth diffeomorphisms $q$ of the circle satisfying the condition $q(0)=0$. It is quite evident (see 4.4) that the measures $\mu_{s}$ on $\mathbb{L}^{0}(S O(n))$ are Diff ${ }_{0}^{\infty}$-quasi-invariant.

I think that $\mu_{s}$ is Diff ${ }^{\infty}$-quasi-invariant, but I couldn't prove it. In any case there is no problem to construct Diff ${ }^{\infty}$-quasi-invariant measure on $\mathbb{L}^{0}(S O(n))$. Let us consider the automorphism $r_{\theta}: g(\varphi) \rightarrow g(\varphi+\theta)$ of the group $\mathbb{L}^{0}(S O(n))$. Let $\mu_{s}^{\theta}$ be the image 
of the measure $\mu_{s}$ with respect to the automorphism $r_{\theta}$. It is easy to see that the measure

$$
\tilde{\mu}_{s}=\int_{0}^{2 \pi} \mu_{s}^{\theta} d \theta
$$

is $\operatorname{Diff}^{\infty} \ltimes \mathbb{L}^{\infty}(S O(n))$-quasi-invariant.

\subsection{Two-Side Quasi-Invariant Measures on Groups}

Proposition 5.1. Let $G$ be a group and $H$ be a subgroup. Let us consider a left $G$-quasi-invariant measure $\mu(g)$ on $G$. Then the convolution of the measures $\mu(g) * \mu(g)$ is two-side $H$-quasi-invariant.

Proof. Evident.

(I am grateful to A. M. Vershik who told me this remark.)

For this reason we have the possibility to obtain two-side Diff $^{\infty}$-quasi-invariant measures on the group Diff ${ }^{\infty}$ and two-side $\mathbb{L}^{\infty}(S O(n))$-quasi-invariant measures on $\mathbb{L}^{0}(S O(n))$.

\subsection{Measures on Loops on Homogeneous Spaces}

Let $\mu$ be a two-side $\mathbb{L}^{\infty}(S O(n))$-quasi-invariant measure on $\mathbb{L}^{0}(S O(n))$. Let $H$ be a subgroup in $S O(n)$. Consider the projection $S O(n) \rightarrow S O(n) / H$. Then we have the projection $\mathbb{L}^{0}(S O(n))$ to $\mathbb{L}^{0}\left(S O(N) / H\right.$ and hence we have a $\mathbb{L}^{\infty}(S O(n))$-quasiinvariant measure on $\mathbb{L}^{0}(S O(n) / H)$.

\subsection{On Shavgulidze Measures}

Shavgulidze constructed a family of quasiinvariant measures on the groups of diffeomorphisms (see [N6], [Kh], [Sha]). The relations of Shavgulidze measures and our constructions are not known.

\section{Comments (Representation Theory)}

\subsection{Highest Weight Representations of Diff ${ }^{\infty}$}

Let $H$ be complex Hilbert space. Let us consider $H$ as a real Hilbert space $H_{\mathbb{R}}$. By definition the group $S P U(H)$ consist of real-linear operators in $H_{\mathbb{R}}$ of the form $A=U(1+T)$ where $U$ is unitary (complex-linear) operator and $T$ is Hilbert-Schmidt 
operator. It is clear that $S P U(H)$ is the subgroup in $G L O\left(H_{\mathbb{R}}\right)$. Let us consider the space $\tilde{W}^{1 / 2}$ constructed in Sect. 3.4. Let us consider Hilbert transformation in $\tilde{W}^{1 / 2}$ given by formula

$$
I f(\varphi)=\frac{1}{\pi} \int_{0}^{2 \pi} \operatorname{ctg}\left(\frac{\varphi-\psi}{2}\right) f(\psi) d \psi
$$

It is easy to see that $I$ is orthogonal operator in real space $\tilde{W}^{-1}$ and $I^{2}=-1$. Let us introduce complex structure to $\tilde{W}^{1}$, by definition the operator $I$ is multiplication on imagine unite.

Theorem 6.1 (see [PS], [N2, N6]). Let $q \in \operatorname{Diff}^{\infty}$. Then $T_{1}(q) \in S P U\left(\tilde{W}^{1 / 2}\right)$.

The group $\operatorname{SPU}(\cdot)$ is the classical group of automorphisms of the canonical commutation relation; this group has well-known representation in the bosonic Fock space (so-called "Weil representation" (see, for instance [N6])). The restriction of "Weil representation" to Diff ${ }^{\infty}$ gives highest weight representations of the group Diff $^{\infty}$. Small variation of this construction gives all highest weight representations of Diff $^{\infty}$, see [N6].

Remark. Let $\varrho$ be the highest weight representation of Diff ${ }^{\infty}$ constructed above. Let $\pi$ be the natural representation of Diff ${ }^{\infty}$ in $L^{2}\left(\hat{\tilde{W}}^{1 / 2}\right)$. Then

$$
\pi=\varrho \otimes \varrho^{*}
$$

where $\varrho^{*}$ is a contragradient representation.

\subsection{Almost Invariant Structures}

The constructions of Sect. 2 and construction 6.1 are special cases of "almost invariant structures" [N1-N6].

Olshanskii [O1, O2] discovered some natural family of infinite dimensional analogues of classical groups (so-called " $(G, K)$-pairs”) and constructed many representations of such groups.

As was shown in [N1-N6] there exist many embedding of the groups Diff ${ }^{\infty}$ and $\mathbb{L}^{\infty}(K)$ to Olshanskii's $(G, K)$-pairs, those embeddings allow to construct many representations of Diff ${ }^{\infty}$ and $\mathbb{L}^{\infty}(K)$ in bosonic and fermionic Fock spaces.

For some examples of "almost invariant structures," see Sect. 7.

\subsection{Representations of Finite Functional Dimension}

The group Diff ${ }^{\infty}$ has actions on finite dimensional spaces (for instance on $S^{1}$, on $S^{1} \times S^{1}$, on tangent bundle $T^{*} S^{1}$, etc.), and hence it has unitary representations in functional spaces on finite dimensional manifolds (for a description of such constructions, see [Ki]). In some exceptional points "almost invariant structures" give direct sums of representations of finite functional dimension (see, for instance the case $s=0$ in Sect. 3).

It is interesting to notice that "almost invariant structures" Sects. 2-3 connect highest weight constructions $(s=1 / 2)$ and "trivial" construction $(s=0)$. 


\subsection{Ismagilov's Construction}

Section 3.9 gives untrivial examples for the theory developed in the paper [I].

\subsection{Energy Representations}

It is quite clear that the "energy representations" of loop groups (see [AHTV]) are related to our construction in the point $s=1$.

\section{Actions of Diff ${ }^{\infty}$ and $\mathbb{L}^{\infty}(S O(n))$ on White Noise}

\subsection{The Group $U O(H \oplus i H)$}

Let $H$ be a real Hilbert space. Let $H_{\mathbb{C}}=H \oplus i H$ be the complexification of $H$. Denote by $U O(H \otimes i H)$ the group of operators

$$
g=\left(\begin{array}{cc}
K & L \\
M & N
\end{array}\right): H \oplus i H \rightarrow H \oplus i H
$$

satisfying the conditions

1. $g$ is complex linear (i.e. $K=\bar{N}, M=-\bar{L}$ ).

2. $L=-M$ is Hilbert-Schmidt operator.

The group $U O(H \oplus i H)$ contains the subgroup $O(H)$. This subgroup consist of matrices $\left(\begin{array}{cc}K & 0 \\ 0 & K\end{array}\right)$.

\subsection{Embeddings of $\operatorname{Diff}^{\infty}$ to $U O(\cdot)$}

Let $s \in \mathbb{R}, s \neq 0$. Let us define the integral operator $J_{s}$ in the complex space $L^{2}\left(S^{1}\right)$ by the formula

$$
J_{s} f(\varphi)=\lambda \int_{0}^{2 \pi} \frac{\overline{f(\psi)} d \psi}{\left|\sin \left(\frac{\varphi-\psi}{2}\right)\right|^{1+2 i s}}
$$

where $\lambda$ is defined by the condition

$$
J_{s} \cdot 1=1
$$

It is easy to check that

$$
J_{s}^{2}=1 .
$$

Let us define real subspaces $H_{s}^{ \pm} \subset L^{2}\left(S^{1}\right)$ :

$$
f \in H_{s}^{ \pm} \Leftrightarrow J_{s} f= \pm f .
$$

The condition $J_{s}^{2}=1$ implies $L^{2}\left(S^{1}\right)=H_{s}^{+} \oplus H_{s}^{-}$. Observe next that

$$
J_{s}(i f)=-i J_{s} f \text {. }
$$

Hence $f \in H_{s}^{ \pm}$implies if $\in H_{s}^{\mp}$. Thus the space $L^{2}\left(S^{1}\right)$ can be looked upon as the complexification $\left(H_{s}^{+}\right)_{\mathbb{C}}$ of the space $H_{s}^{+}$. Now let the group Diff ${ }^{\infty}$ acts in $L^{2}\left(S^{1}\right) \cong\left(H_{s}^{+}\right)_{\mathbb{C}}=H_{s}^{+} \oplus i H_{s}^{+}$by the transformations

$$
T_{i s}(s) f(\varphi)=f(q(\varphi)) q^{\prime}(\varphi)^{1 / 2+\imath s} .
$$

Theorem 7.1 (see [N6]).

$$
T_{i s}(q) \in U O\left(H_{s}^{+} \oplus i H_{s}^{+}\right)
$$




\subsection{Embeddings of the Group $\mathbb{L}^{\infty}(S O(n))$ to $U O(\cdot)$}

Let $L^{2}\left(S^{1}, \mathbb{C}^{n}\right)$ be the space of $L^{2}$-functions $S^{1} \rightarrow \mathbb{C}^{n}$. Let us define operator $J_{s}$ in $L^{2}\left(S^{1}, \mathbb{C}^{n}\right)$ by the same formula (7.1). Let us define real subspaces $H_{s, n}^{ \pm} \subset L^{2}\left(S^{1}, \mathbb{C}^{n}\right)$ by

$$
f \in H_{s, n}^{ \pm} \Leftrightarrow J_{s} f= \pm f
$$

Let the group $\mathbb{L}^{\infty}(S O(n))$ acts in $L^{2}\left(S^{1}, \mathbb{C}^{n}\right) \cong\left(H_{s}^{+}\right)_{\mathbb{C}}$ by the usual way

$$
Q(g(\varphi)) f(\varphi)=g(\varphi) f(\varphi) \text {. }
$$

Theorem 7.2.

$$
Q(g(\varphi)) \in U O\left(H_{s, n}^{+} \oplus i H_{s, n}^{+}\right)
$$

\subsection{Embeddings $U O(H \oplus i H)$ to $G L O(H \oplus i H)$}

Now we want to construct series of embeddings $U O(H \oplus i H)$ to the group $G L O(H \oplus i H)$ of space $H \oplus i H$ (the space $H \oplus i H$ is looked upon as real Hilbert space). Thus we will obtain quasi-invariant actions of the group $U O(H \oplus i H)$ in $\widehat{H \oplus i} H$, and hence we will obtain quasi-invariant actions of the groups Diff ${ }^{\infty}$ and $\mathbb{L}^{\infty}(S O(n))$.

Let

$$
\left(\begin{array}{cc}
K & L \\
-\bar{L} & \bar{K}
\end{array}\right): H \oplus i H \rightarrow H \oplus i H
$$

be an element of $U O(H \oplus i H)$.

Fix $\kappa \in \mathbb{R}$, let

$$
\begin{aligned}
\tau_{\kappa}\left(\begin{array}{cc}
K & L \\
-L & K
\end{array}\right)= & \left(\begin{array}{cc}
\cosh \kappa & \sinh \kappa \\
\sinh \kappa & \cosh \kappa
\end{array}\right)^{-1}\left(\begin{array}{cc}
K & L \\
-\bar{L} & \bar{K}
\end{array}\right) \\
& \times\left(\begin{array}{cc}
\cosh \kappa & \sinh \kappa \\
\sinh \kappa & \cosh \kappa
\end{array}\right) .
\end{aligned}
$$

It is easy to see that $\tau_{\kappa}$ is the embedding $U O(H \oplus i H)$ to the group $G L O(H \oplus i H)$. In the case $\kappa=0$ we have the non-interesting embedding $U O(H \oplus i H) \rightarrow O(H \oplus i H) \subset$ $G L O(H \oplus i H)$, but in other cases this construction give untrivial representations of $U O(\cdot)$.

\subsection{Affine Action of $U O(H \oplus i H)$}

Let $Z$ be the space of self-adjoint real-linear Hilbert-Schmidt operators

$$
T=\left(\begin{array}{cc}
A & B \\
C & \mathscr{D}
\end{array}\right): H \oplus i H \rightarrow H \oplus i H .
$$

The space $Z$ is the Hilbert space with respect to the scalar product

$$
\left\langle T_{1}, T_{2}\right\rangle=\operatorname{tr}\left(T_{1} T_{2}\right)
$$

Let the group $U O(H \oplus i H)$ act on the space $Z$ by the affine transformations

$$
\sigma(g) T=g^{t} T g+g^{t} g-E,
$$


where $g \in U O(H \oplus i H), T \in Z$. The transformations $T \rightarrow g^{t} T g$ are orthogonal in $Z$ and $g^{t} g-E \in Z$. Hence (see Sect. 1.3) the Gauss measure in the canonical extension $\hat{Z}$ of $Z$ is quasi-invariant with respect to transformations $\sigma(g)$.

Thus we obtained the quasi-invariant action of the group $U O(H \oplus i H)$ (and hence $\operatorname{Diff}^{\infty}$ and $\mathbb{L}^{\infty}(S O(n))$ in $\hat{Z}$.

\subsection{Comments}

A. By definition white noise is the space $\widehat{L^{2}}$. Thus we constructed in Sects. 7.2-7.4 the series of quasi-invariant actions of Diff ${ }^{\infty}$ and $\mathbb{L}^{\infty}(S O(n))$ on the sum of two copies of white noise.

B. Analogous constructions exist for all "compact" almost invariant structures, see $[\mathrm{N6}, \mathrm{O} 2]$. Compact almost invariant structures also give actions of Diff ${ }^{\infty}$ and $\mathbb{L}^{\infty}(K)$ in fermionic Fock spaces.

Acknowledgements. I am very grateful to A. M. Vershik, P. Malliavin, R. S. Ismagilov, G. I. Olshanskii for discussions of this subject.

\section{References}

[AHTV] Albeverio, S., Hoegh-Krohn, R., Testard, D., Vershik, A.M.: Factorial representations of path groups. J. Funct. Anal. 51, 115-131 (1983)

[GGV] Gelfand, I.M., Graev, M.I., Vilenkin, N.Ya.: Generalized functions, Vol. 5. Moscow: Fizmatgiz 1962, English transl.: New York: Academic Press 1966

[I] Ismagilov, R.S.: Unitary representations of the group of diffeomorphisms of the circle. Funct. Anal. Appl. 5, 209-216 (1971)

[Ka] Kac, V.G.: Infinite dimensional Lie algebras. Boston: Birkhäuser 1983

[Kh] Khafizov, M.U.: A quasiinvariant smooth measure on the diffeomorphisms group of a domain. Math. Notes 48, 968-972 (1990)

[Ki] Kirillov, A.A.: On unitary representations of groups of diffeomorphisms and some its subgrops. Preprint IPM, Moscow, 1974, N62, (Russian). English transl. in Selecta Sov. Math. 1, 351-372 (1981)

[Ku] Kuo, Hui-Hsuing: Gauss measures in Banach spaces. Lect. Notes Math. 463 (1975)

[L] Loeve, M.: Probability theory, Vol. 2, 4 ed. Berlin, Heidelberg, New York: Springer 1978

[MM] Malliavin, M.P., Malliavin, P.: Integration on loop groups. I. J. Funct. Anal. 93, 207-237 (1990)

[N1] Neretin, Yu.A.: Complementary series of representations of the group of diffeomorphisms of the circle. Russ. Math. Surv. 37, 229-230 (1983)

[N2] Neretin, Yu.A.: Bosonic representations of the group of diffeomorphisms of the circle. Sov. Math. Dokl. 2, 411-414 (1983)

[N3] Neretin, Yu.A.: Unitary representations with highest weight of the group of diffeomorphisms of a circle. Funct. Anal. Appl. 17, 235-236 (1983)

[N4] Neretin, Yu.A.: Almost invariant structures and constructions of representations of the group of diffeomorphisms of the circle. Sov. Math. Dokl. 35, 500-503 (1983)

[N5] Neretin, Yu.A.: Almost invariant structures and related representations of the group of diffeomorphisms of the circle. Representations of Lie groups and related topics. Zhelobenko, D.P., Vershik, A.M. (eds.), New York: Gordon and Breach 1991, pp. 245268

[N6] Neretin, Yu.A.: Representations of the Virasoro algebra and affine algebras. In: Kirillov, A.A., Neretin, Yu.A. (eds.), Noncommutative harmonic analysis I. Sovr. probl. matem. Fund. Napravl. 22, 164-225 (1988), in Russian. English transl. to appear in Encyclopaedia of Math. Sci., vol. 22 
[N7] Neretin, Yu.A.: Mantles, trains and representations of infinite dimensional groups. Proceedings of First European Math. Congress, Birkhäuser, to appear

[O1] Olshanskii, G.I.: Unitary representations of infinite dimensional classical pairs $(G, K)$ and the formalism of R. Howe. Sov. Math. Dokl. 27, 294-298 (1983)

[O2] Olshanskii, G.I.: Unitary representations of infinite dimensional pairs $(G, K)$ and the formalism of R. Howe. Representations of Lie groups and related topics. Zhelobenko, D.P., Vershik, A.M. (eds.), New York: Gordon and Breach 1991, pp. 269-464

[PS] Pressley, A., Segal, Gr.: Loop groups. Oxford: Clarendon Press 1986

[Re] Repka, J.: On tensor products of unitary representations of $S L_{2}(R)$. Am. J. Math. 100, 747-774 (1978)

[Ro] Rohlin, V.A.: On fundamental ideas of measure theory. Math. Sbornik 25(67), 107-150 (1949). English transl. in Am. Math. Soc. Trans. Ser. 1, Vol. 10, 1-54

[Sha] Shavgulidze, E.T.: On example of the measure quasiinvariant relative to action of the group of diffeomorphisms of finite dimensional manifold. Sov. Math. Dokl. 38, 622-625 (1988)

[ShF] Shilov, G.E., Fan Dyk Tin: Integral, measure and derivative in linear space. Moscow: Nauka 1967 (Russian)

[T] Treves, F.: Introduction to pseudo-differential and Fourier integral operators. Vol. 1. New York: Plenum Press 1980

[V] Vershik, A.M.: On description of invariant actions of some infinite dimensional groups. Sov. Math. Dokl. 15, 1396-1400 (1974)

Communicated by Ya. G. Sinai 